\title{
Ultra-low Power Sensors with Near Field Communication for Mobile Applications
}

\author{
Esko Strömmer ${ }^{1}$, Mika Hillukkala ${ }^{1}$, Arto Ylisaukko-oja ${ }^{1}$ \\ ${ }^{1}$ VTT, P.O. Box 1100, FI-90571 Oulu, Finland \\ E-mail: Firstname.Surname@vtt.fi
}

\begin{abstract}
In this paper, we study the applicability of an emerging RFID-based communication technology, NFC (Near Field Communication), to ultra-low power wireless sensors. We present potential application examples of passive and semi-passive NFC-enabled sensors. We compare their NFC-based implementations to corresponding implementations based on short-range radios such as Bluetooth and Wibree. The comparison addresses both technical properties and usability. We also introduce Smart NFC Interface, which is our general-purpose prototype platform for rapid prototyping of NFC and Bluetooth implementations. Two pilot sensor implementations and an NFC-Bluetooth gateway implementation based on our platform are presented and evaluated. Finally, needs and possibilities for technical improvements of available NFC technology are discussed.
\end{abstract}

Keywords: Near field communication (NFC), Mobile applications, Wireless sensors

\section{Introduction}

Sensors with inductive data transmission and powering have been under research activities for several years. Examples can be found in [1], [2] and [3]. Proposed solutions have usually been based on proprietary technologies or standard RFID technologies. A problem concerning commercial mobile sensor applications has been the lack of standardized inductive technology in mobile handsets such as mobile phones and PDAs, which has made a specific reader device or add-on module mandatory.

In March 2004, a new interconnection technology, Near Field Communication (NFC), was launched by Sony, Philips and Nokia, by the establishment of the NFC Forum. The NFC Forum is a non-profit industry association for advancing the use of NFC short-range wireless interaction in consumer electronics, mobile devices and PCs. In co-operation with ECMA, the NFC Forum will promote the implementation and standardization of NFC technology to ensure interoperability between devices and services. As of the end of 2006, about 100 members have joined the NFC Forum. Within the next few years, NFC is forecast to penetrate commercial mobile phones [4].

We expect NFC to provide a potential solution for enhancing existing and emerging mobile applications with data acquisition from various sensors. NFC 
supports the use of mobile handsets by touch-based interaction, which is an intuitive and user-friendly way of establishing connections and exchanging information between mobile handsets and other devices [5]. From the sensor application viewpoint, NFC can provide easy-to-use touch-based access to the sensor data by low-cost mobile phones and other mobile handsets, which will make the application specific reader device unnecessary and thus decrease the system level costs dramatically. As NFC is capable of transferring power between devices, fundamentally NFC enables semi-passive implementation of sensors with multimonth to multi-year battery lifetime and even passive implementation without any power source. However, this calls for commercial NFC technology that supports zeropower operation of the NFC transceiver (similar to an RFID tag) when waiting for activation from an active NFC device, and efficient power management during communication.

\section{Near Field Communication Technology}

Near field communication (NFC) is a very short-range (max. $20 \mathrm{~cm}$, but typically only a few $\mathrm{cm}$ ), wireless point-to-point interconnection technology, evolved from a combination of earlier RFID contactless identification and interconnection technologies (ISO14443A/MIFARE/FeliCa). It enables users of handheld electronic devices to access content and services in an intuitive way by simply "touching" smart objects (e.g. sensors, RFID tags, other handheld devices), i.e. connecting devices just by holding them next to each other. The communication is based on inductive coupling. The $13.56 \mathrm{MHz}$ carrier frequency is used and the available data rates are 106, 212 and $424 \mathrm{kbps}$. The related standards are shown in Fig. 1.

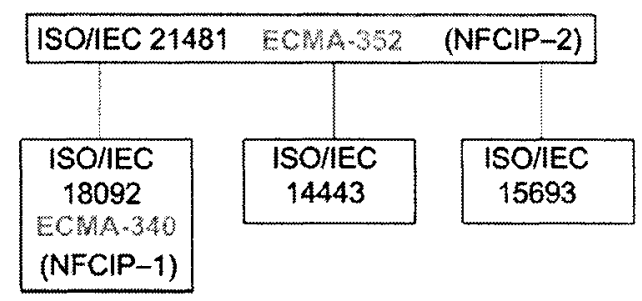

Fig. 1. NFC-related standards. The upper layer defines the mechanism of selecting the communication mode on the lower layer [6].

The legacy of earlier standards gives NFC compatibility benefits with existing RFID applications, such as access control or public transport ticketing - it is often possible to operate with old infrastructure, even if the RFID card or reader is replaced with an NFC-enabled mobile phone, for example. This is possible because of NFC's capability to emulate both RFID readers ("reader/writer mode") and RFID tags ("card emulation mode"). NFC hardware can include a secure element for improved security 
in critical applications such as payments. For example, a credit card could be integrated into a mobile phone and used by contactless credit card readers over NFC.

In addition to the reader/writer and card emulation modes, there is an NFC-specific NFCIP-1 mode ("peer-to-peer mode"), defined in the ECMA-340 standard. This mode is intended for peer-to-peer data communication between devices. In this mode, NFC is comparable to other short-range communication technologies such as Bluetooth, Wibree and IrDA, although the physical data transfer mechanism is different. In this respect, NFC can be seen as a rival of these technologies, even though it can also complement them. NFC can open a connection between two devices that are brought close to each other, and the actual communication will then occur by a longer range technology such as Bluetooth.

In peer-to-peer mode, the participant that starts the communication is called the initiator and the other participant the target. The peer-to-peer mode is divided into two variants: active mode and passive mode. In active mode, both participants generate their own carrier while transmitting data. In passive mode, only the initiator generates a carrier during communications, and the target device uses load modulation when communicating back to the initiator, in a way similar to passive RFID tag behavior [7]. This makes it possible to save power in the target device, which is a useful feature if the target device has a very restricted energy source, such as a small battery. Fundamentally it is possible to make a target device operate for several years without battery replacement or even without any battery by the power that is emitted by the initiator.

NFC is a standard technology that has recently achieved commercial availability via NFC chips, modules, mobile phones and PDAs. NFC is also backed by the leading mobile phone manufacturers and its deployment and chip development will be strongly driven via its integration into cellular handsets. According to the downgraded forecast of the NFC-enabled mobile handsets by ABI Research in September 2006, the number of shipped NFC-enabled handsets in 2011 will be 450 million units, or 30 per cent of all handsets.

The most natural choice for NFC-enabled sensors is the passive peer-to-peer (NFCIP-1) communication mode. Also the card emulation mode is feasible.

\section{Application Scenarios}

In this chapter we present some application scenarios of sensors with NFC connectivity. We point out that these are not yet commercial products but only examples of the versatile application possibilities of NFC-enabled sensors. Our scenarios are divided into three main groups:

- passive (also batteryless) sensors,

- user controlled semi-passive (also semi-active or battery assisted) sensors, and

- stand-alone semi-passive sensors for long-term monitoring. 


\subsection{Passive Sensors}

A fundamental operation principle of RFID systems is that the passive end of the communication link (tag) gets all its energy for operation from the field generated by the active end of the communication link (reader). It is also possible to apply this principle in the implementation of NFC-enabled passive sensors by replacing the RFID reader with an NFC device that operates in reader-writer or in peer-to-peer initiator mode, and the tag by an NFC-enabled sensor that operates in card emulation or in peer-to-peer passive target mode. We have estimated that at least several milliwatts of power can be delivered to an NFC antenna that has a diameter of $25 \mathrm{~mm}$ with field strengths defined in the associated NFC standard $(1.5-7.5 \mathrm{~A} / \mathrm{m})$. With larger antennas, the delivered energy can be higher. If the transferred power is not enough for the sensor due to, e.g. a smaller antenna or longer range, it is possible to add a powering capacitor into the sensor module that is charged before the measurement and from which power is taken during the measurement. Because battery replacement or wires are not required, these sensors can be easily embedded in various structures (e.g. walls, injection molded products) during their manufacturing.

An example of a low power sensor that could easily be made NFC-enabled is a silicon based temperature sensor such as LM20 [8]. The current consumption of this sensor is only $10 \mu \mathrm{A}$, thus only a minor part of the typical power transferred over an NFC link. An A/D-converter and some logic are needed between the temperature sensor and the NFC transceiver. This can be implemented separately or included in the same microcontroller that takes care of the NFC protocol. Another example is a moisture sensor that could be embedded into walls and flooring of the house close to water pipes or floor drains for indicating possible leaks that usually cannot be detected outside the structure until they have caused severe damages to the structure.

\subsection{User-Controlled Semi-passive Sensors}

Semi-passive (also semi-active or battery assisted) RFID tags have an on-board power source (for example, a battery) and electronics for performing specialized tasks. The on-board power supply provides energy to the tag for its operation. However, for transmitting its data, a semi-passive tag uses power that is emitted by the reader in the same manner as passive tags [9]. Instead of a battery, a parasitic power supply exploiting ambient energy such as temperature gradients, light, mechanical forces and movements or electromagnetic fields from other sources than the reader are possible.

The same operation principle could also be applied to NFC-enabled sensors, in which case we call them semi-passive sensors. User-controlled semi-passive sensors are in a non-operating state most of the time, a state in which their current drain from the on-board battery is negligible. The sensor is activated by some action from the user. This can be pushing a switch-on button or bringing an active NFC-enabled device to the vicinity of the sensor, in which case its NFC transceiver that operates in card emulation or in peer-to-peer passive target mode activates the entire sensor.

User-controlled semi-passive sensors could be well used, for example, in health or disease management in which monitoring is based on sparse measurements (e.g. blood pressure, weight). In these kinds of applications, the amount of data is typically 
limited. The sensor is switched on and the measurement activated by the user. Measurement results could be transferred to an NFC-enabled mobile phone either immediately or later in blocks of several results acquired over time. Scenarios of this kind are discussed more closely in [10]. Another potential application could be a battery assisted implanted blood glucose sensor such as in [11] with activation and communication by NFC instead of proprietary radio technology. However, due to the very limited size of implant sensors and thus also very small maximum antenna size, the technical feasibility of NFC-enabled implant sensors requires further investigation, and the maximum communication range will in any case be much shorter than in the other examples mentioned above.

\subsection{Stand-Alone Semi-passive Sensors for Long-Term Monitoring}

Semi-passive sensors could also be applied to various autonomous long-term monitoring applications. In addition to user actions, these sensors have to be able to be activated by other external events or periodically by an internal real-time clock or other wakeup timer. This will make them a little more complicated than the user controlled sensors, but still technically feasible.

As an example, an NFC-enabled condition monitoring sensor could be fitted onto goods that require special care during transport. At the reception end, the sensor is checked by an NFC-enabled reader device that gives an alarm if something unexpected has occurred during the transport. Another application area could be longterm physical activity and health monitoring. For example, an inertial sensor integrated into a shoe could measure the daily walking distance, and the result could be read from the sensor at the end of the day by an NFC-enabled mobile phone. A potential application example is also an RFID tag size electric supply meter that could be integrated into any electrical appliance and could take its supply power from its mains supply capacitively. Again, the cumulated energy consumption of the appliance could be read by an NFC-enabled mobile phone.

\section{NFC vs. Short-Range Radios}

The nature of NFC-based wireless sensors clearly differs from many conventional implementations of wireless sensors and sensor networks. In short-range radio based wireless sensor networks, there is not necessarily a human user at all, but the sensors may serve as a part of an automation system, for example. In contrast, a human user plays a central role in typical NFC-based implementations, a mobile handset such as a mobile phone being the typical tool for the interaction.

Bluetooth enabled phones are currently widely available. However, according to [4], also NFC should become a common technology in mobile phones in the near future. Comparing these technologies is thus relevant. Fundamentally the NFC communication offers the following advantages over Bluetooth: 
- NFC enables intuitive, easy-to-use touch-based communication and interaction between two devices.

- Communication set-up latency with NFC is typically some hundreds of milliseconds, whereas with Bluetooth it is typically several seconds.

- NFC enables longer lifetime of the sensor battery, or even batteryless implementation of the sensor.

- Pure NFC communication enables lower price, since NFC is technically less complex than Bluetooth.

- Due to its shorter range and near field coupling, NFC is more immune to eavesdropping and intentional or unintentional interferences.

The very short communication range of NFC could be seen as a mere shortcoming, but the matter is not that simple. From the user point-of-view, the association between communicating devices is very intuitive with NFC: to start the communication, just "touch" a device with the mobile handset, instead of trying to identify the correct device from a potentially long menu in the mobile handset, as in the case of Bluetooth communication. This type of physical browsing user paradigm may prove to be a very powerful one from the human-computer interaction perspective [5]. In an ideal situation, no key presses or browsing of menus is required, or at least this is kept to a minimum. For example, confirmation by a key press can sometimes be necessary for security reasons.

Still, communication range is the main limitation of NFC compared to Bluetooth. This, depending on the application, can create following disadvantages:

- NFC does not suit portable devices that require online connectivity to another portable device or to a fixed access point.

- Lower bit rate together with the short communication range can make the touchbased transfer of longer data blocks unpleasant.

- The placement of the antenna is more critical. The location of the antenna has to be indicated to the user.

These disadvantages can be partly overcome by combining NFC with Bluetooth or WLAN, which on the other hand will mean that some of the advantages such as the lower price of pure NFC implementation are lost. Still, this is an important aspect in NFC application possibilities. In semi-passive applications requiring transmissions of longer data blocks, NFC may be used only to initiate the connection, and the actual data transmission is then performed by means of Bluetooth or WLAN which offer higher data rates and longer communication range. In that case, NFC is a tool to configure the connection easily and explicitly with a simple touch, instead of configuring the connection by performing device searches or setting of configuration parameters by browsing menus and using keypads. Compared to pure NFC, the user does not need to keep the devices close to each other after the connection has first been initiated. In a similar way, NFC can also be used to configure long-term device pairs (such as Bluetooth trusted pairs).

A classic problem with wireless sensors is the source of power. A reasonable solution is essential for the overall usability of the system. Otherwise the maintenance of the system may require too much effort. Good examples of such cases are the 
implantable and wall-embedded sensors mentioned in Chapter 3, but this easily becomes an issue even in far more conventional scenarios. The trend is to decrease the power consumption of radios to enable longer operation time from small batteries (Bluetooth derived Wibree technology, for example [12]). However, this only makes the maintenance interval longer instead of eliminating the need for maintenance completely. Basically the only way to avoid maintenance for energy supply in case of short-range radios would be to apply solutions like harvesting power from ambient energy sources such as light, temperature gradients, vibration and so on. Unfortunately, this method tends to increase complexity and price, and requires very case-specific designs.

Here NFC (or RFID) based implementations have the potential of being superior to other currently available technology alternatives: even fully passive implementations are possible with some NFC chips such as Microread by Inside Contactless [13], getting the required energy for both the measurement (and/or a secure element) and communications from the reading device. This enables potentially maintenance-free devices.

If there is no energy storage, the restriction is that the measurement can only take place while the reader is nearby. In some applications (see Chapter 3.3), a semipassive implementation is needed instead to enable measurements also at other times. Even then, the average current consumption of an NFC-enabled semi-passive sensor can be on the order of tens of microamperes without sacrificing the communication latency - which typically needs to be sacrificed when optimizing power in the case of other short-range radio technologies.

\section{Pilot Demonstrators}

\subsection{Smart NFC Interface}

Smart NFC Interface is a multi-purpose platform that we have developed for evaluating the NFC technology, rapid prototyping and demonstrating of related product ideas, and fastening the associated product development by technology transfer. It is a light, matchbox-sized device with a microcontroller, rechargeable LiIon battery with charging electronics, data logging memory, RS-232 serial port and other wired communications, as well as NFC, IrDA and Bluetooth wireless communications. The structure is modular to facilitate modifiability for different applications. There are connectors for extensions such as sensors. An LM20 temperature sensor [8] is readily included, and the design supports ultra-low power operating modes. However, passive (batteryless) sensors are not yet supported. The microcontroller is programmable by $\mathrm{C}$. A photograph of the electronics and the basic application scenario in sensor applications is shown in Fig. 2. 

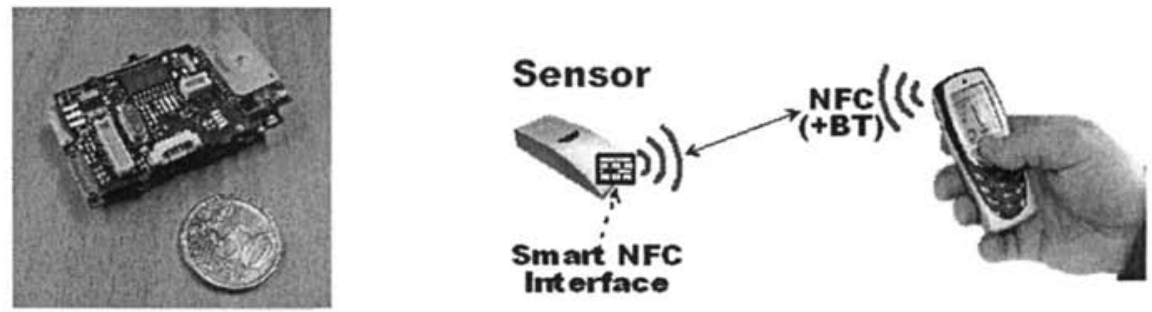

Fig. 2. Smart NFC Interface module (Communication Board on top, NFC antenna not installed) and its basic application scenario as a sensor interfacing module.

The block diagram of the Smart NFC Interface electronics is shown in Fig. 3. In its basic form, the device contains two boards: 1) Basic Board, and 2) Communication Board. The dimensions of both boards are $56 \times 31 \mathrm{~mm}$. Overall thickness of the device including the default rechargeable battery is $16 \mathrm{~mm}$ without encapsulation (boards attached one on top of another).

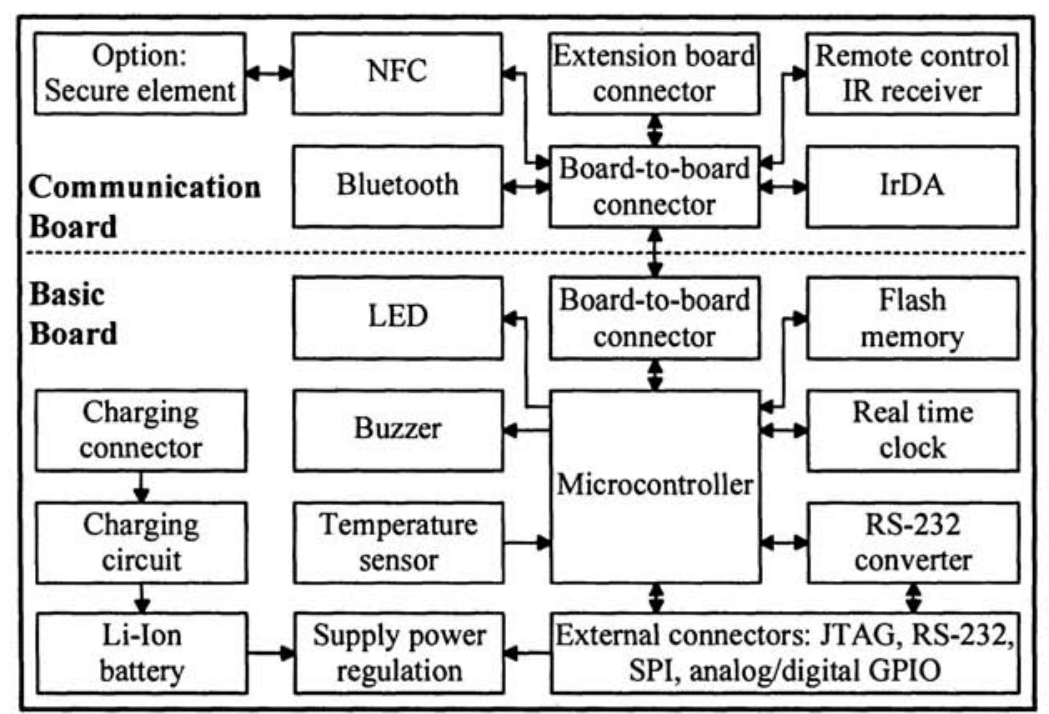

Fig. 3. Block diagram of the Smart NFC Interface electronics

The Smart NFC Interface has a single 8-bit microcontroller (Atmel AVR series ATmega128L). The microcontroller is equipped with 128 kilobytes of flash program memory that enables easy reprogramming and debugging via a JTAG interface, 4 kilobytes of internal RAM and 4 kilobytes of EEPROM. An external $8 \mathrm{Mbit}$ flash memory is connected to the SPI bus of the microcontroller. This can be used for data logging purposes in stand-alone semi-passive sensors (see Chapter 3.3), for example. 
The Smart NFC Interface is equipped with a Philips PCF8563T real-time clock (RTC) circuit, whose main purposes are 1) enabling recording of timestamps for events, and 2) serving as a wakeup timer, thus facilitating ultra-low power modes. Both of these are particularly useful features in stand-alone semi-passive sensor implementations for long-term monitoring according to Chapter 3.3.

A versatile power supply circuitry is used, enabling power to be taken not only from the internal battery, but also from RS-232 serial port or other external supplies. The power supply automatically adjusts to a wide range of input voltages. The charging circuit is connected to the LED, which indicates the charging status. While not charging, the LED is also software controlled by the microcontroller. Another supported UI feature is a buzzer.

On the Communication Board, the NFC transceiver is based on NXP Semiconductor PN531 chip, which is connected to the microcontroller via its SPI bus. A SmartMX secure chip can optionally be installed. Bluetooth (Class 2) is based on Bluegiga WT12-A-AI module, which contains Bluetooth protocol layers up to RFCOMM and profiles, and is connected to the microcontroller by UART. IrDA support currently exists only in hardware, based on Microchip MCP2150-I/SO IrDA controller and Zilog ZHX1403 IrDA transceiver. There is also hardware support for a remote control infrared (IR) receiver.

The general purpose IO (GPIO) on the external connector includes an analog input. This can be used to connect sensors. Digital interfaces such as RS-232/UART and SPI are also included. RS-232 levels are converted to logic level UART and vice versa by Maxim MAX3222CAP.

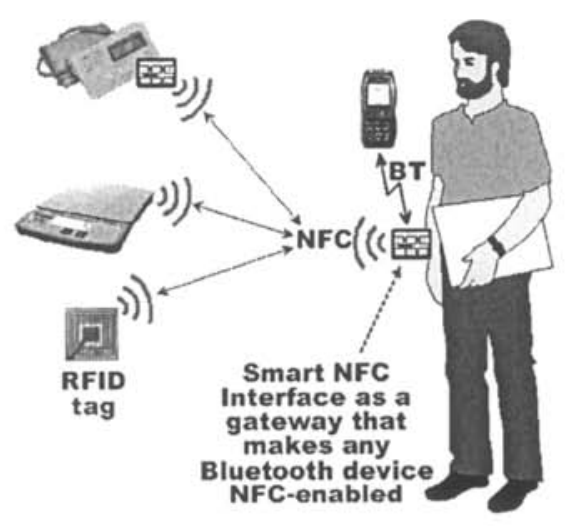

Fig. 4. Applying the Smart NFC Interface as a gateway

Two pilot NFC sensor implementations based on the Smart NFC Interface are presented in Chapters 5.2 and 5.3. Since the NFC-enabled phones and their JSR-257 contactless communication API standard do not currently support peer-to-peer communication, we have also configured the Smart NFC Interface to operate as a gateway device between non-NFC mobile phones and NFC-enabled sensors (see Fig. 4). The gateway is an active NFC device in peer-to-peer initiator or reader/writer 
mode and is connected to a host device (a mobile phone or a laptop) by Bluetooth. When the gateway detects a target NFC device or an RFID tag, it opens the NFC communication, reads the data from the target and sends the data to the host device.

\subsection{Temperature sensor}

The temperature sensor pilot is an example of a user controlled semi-passive sensor according to Chapter 3.2 that is activated by another NFC device. The pilot consists of a Smart NFC Interface module with an inbuilt temperature sensor and an active NFC-enabled mobile device. This can be either an NFC-enabled mobile phone or a Smart NFC Interface module that is configured to gateway mode (see the previous chapter).

At the sensor end, the Smart NFC Interface sets its NFC transceiver to target mode and waits for an active NFC device. When it is detected, the inbuilt temperature sensor is powered up and after a short stabilization time the temperature sensor output is sampled. The result is converted into Celsius degrees and sent by the NFC transceiver as an ASCII character string.

The gateway sets its NFC transceiver to poll target NFC devices in initiator mode. After detecting the target temperature sensor, it sends a command via the NFC communication link to it and waits for a reply. After receiving the ASCII character string from the temperature sensor, it sends it to the host device via Bluetooth for displaying it to the user.

\subsection{Energy consumption meter}

Another pilot that we have implemented is a slightly modified version of the previous example, being an example of a stand-alone semi-passive sensor for long-term monitoring according to Chapter 3.3. In this pilot, a separate sensor box is connected to the serial port of the Smart NFC Interface. This sensor box monitors electric current inside a mains wire with a tiny coil that is attached on the surface of the wire.

The program flow on the active NFC device (gateway) side is the same as in previous example, but there are a couple of changes on the target side. After a measurement result message is read from the sensor box to the target Smart NFC Interface module, there has to be some sanity checks to make sure that the message is not corrupted. Then the measurement result is extracted from the message structure, calibrated and added to previous results for calculating the cumulated energy consumption. The message to be sent through NFC is constructed by making use of this newest measurement result.

All this data processing takes quite a long time and it is not possible to do these calculations during NFC communication, since the NFC initiator would timeout before all calculations would be finished on the NFC target (sensor) side. Therefore calculations must be carried out before the NFC communication. When an active NFC device is detected, the pre-calculated data is sent to it directly from the memory of the target NFC device. 
The current consumption of the Smart NFC Interface operating in target mode (sensor) is approximately $10 \mathrm{~mA}$ for the host processor and $25 \mathrm{~mA}$ for PN531. It is possible to reduce the average total current consumption (host + PN531) to $3.5 \mathrm{~mA}$ by periodically starting and stopping PN531, and using the RTC to wakeup the host processor from sleep every 50 milliseconds. According to PN531 datasheet [14] it is possible to put PN531 into power down mode where its current consumption is only $30 \mu \mathrm{A}$. In this mode, the combined current consumption during sleep periods would be $40 \mu \mathrm{A}$, and PN531 should be able to detect an active NFC device and wake up the host processor by using its interrupt line. Unfortunately this power down mode does not work with the SPI interface of PN531 that we are using in our design.

The current consumption of the gateway is approximately $10 \mathrm{~mA}$ for host processor and $90 \mathrm{~mA}$ for the NFC chip PN531. With antenna tuning, it is possible to affect the PN531 current consumption, but it will also have an effect on the reading distance. It is also possible to use NFC in short bursts, for example three read cycles per second, reducing the average total current consumption to $15 \mathrm{~mA}$ without sacrificing the short latency time evidently.

The NFC communication data rate in our pilots is $106 \mathrm{kbps}$. It takes a few tens of milliseconds to open an NFC communication channel and send a few bytes of measurement data. This delay is very short compared to delays produced in mobile phone software and user interface.

\section{Discussion}

We conclude that NFC is a high-potential technology for implementing ultra-low power sensors with touch-based communication with mobile handsets. Contrary to other RFID technologies, NFC is backed by the leading mobile phone manufacturers and is expected to penetrate into mobile phones in near future. This will improve the availability of NFC technology in general and facilitate its applicability in mobile sensor applications, too.

However, from the wireless sensor perspective, there are still limitations in commercially available NFC chip and phone implementations. Thus we were not able to pilot all application scenarios that we presented in Chapter 3. For example, the PN531 by NXP Semiconductors supports passive RFID tag type of load modulation in NFC communications, but it does not support taking energy for all operations from the reader side. This prevents implementing passive sensors based on this device. On the other hand, the lowest power consumption of the device with receiver active is 30 $\mu \mathrm{A}$ from 2.5 volts [14]. This is still quite a lot and does not enable coin-cell battery lifetime of several years in semi-passive implementations. We also failed to utilize the full potential of the device, based on giving a wakeup interrupt for an external microcontroller when an NFC initiator comes nearby. This caused a severe compromise to power optimization.

NFC chips are also being offered and developed to support fully passive operation. The main reason for this trend is the target to enable critical NFC functionality, such as access control and payments in mobile phones, even if the battery has been completely discharged. An example of such a device is described in [13]. NFC chips 
having suitable interfaces, support for passive operation and a high degree of integration are also needed for making the implementation of NFC-enabled sensors easier. Good power management support is essential for semi-passive sensors. NFC chips with reduced functionality (e.g. without active modes) could be a reasonable approach to ultra-low power sensors, since this would cut down their power consumption and price further.

The JSR-257 API standard for NFC phones needs to be evolved further to include support for peer-to-peer mode. The range of NFC-enabled mobile handsets is still limited, concerning especially smartphones. This can be partially overcome by an NFC-Bluetooth gateway that we have demonstrated. This kind of approach of having a separate, wireless NFC communication device may be desirable even in the long run in some special applications.

\section{References}

1. Li, Y.L., Liu, J.: A 13.56 MHz RFID Transponder front-end with Merged Load Modulation and Voltage Doubler-clamping Rectifier Circuits. Circuits and Systems. IEEE International Symposium (2005) 5:5095-5098.

2. Chevalerias O., O'Donnell T., Power D., O'Donovan N., Duffy G., Grant G., O'Mathuna S. C.: Inductive Telemetry of Multiple Sensor Modules. IEEE Pervasive Computing, Vol. 4 No. 1 (2005) 46-52

3. Sawan M.: Wireless smart implants dedicated to multichannel monitoring and microstimulation. Proceedings of the IEEE/ACS intemational Conference on Pervasive Services (2004) 21-26

4. Near Field Communications (NFC). Simplifying and Expanding Contactless Commerce, Connectivity, and Content. ABI Research, Oyster Bay, NY (2005)

5. Välkkynen, P. Korhonen, I. Plomp, J., Tuomisto, T., Cluimans, L., Ailisto, H., Seppä, H.: A user interaction paradigm for physical browsing and near-object control based on tags.

Proceedings of Physical Interaction Workshop on Real World User Interfaces, Udine,IT, 811 Sept., 2003. University of Udine, HCI Lab., Department of Mathematics and Computer Science (2003), 31 - 34

6. ECMA International web site (2003). http:/www.ecma-international.org/publications/standards/Ecma-352.htm.

7. ECMA International standard ECMA-340. 2nd Edition (2004). http://www.ecma-international.org/publications/files/ECMA-ST/Ecma-340.pdf.

8. National Semiconductor LM20 web site (2007). http://www.national.com/pf/LM/LM20.html.

9. Lahiri S.: RFID: A Technology Overview. IBM Press (2005). http://www.ibmpressbooks.com/articles/article.asp? $\mathrm{p}=413662 \& \mathrm{rl}=1$.

10.Strömmer E., Kaartinen J., Pärkkä J., Ylisaukko-oja A., Korhonen I.: Application of Near Field Communication for Health Monitoring in Daily life. Proceedings of 28th IEEE EMBS International Conference. New York City, USA (2006) 3246-3249.

11.SMSI Glucose Sensor. Sensors for Medicine and Science, Inc. (2007). http://www.s4ms.com/products_glucose.htm.

12. Wibree technology data sheet. http://www.wibree.com/technology/Wibree_2Pager.pdf

13.Inside Contactless Microread fact sheet. http://www.insidecontactless.com/products/microread-eng.pdf

14.NXP Semiconductors PN531- $\mu$ C based Transmission module Objective Short Form Specification February 2004 Revision 2.0. 